\title{
MODELING OF DYNAMICS OF COOPERATING WHEELED MOBILE ROBOTS
}

\author{
Andrzej Burghardt, Piotr Gierlak, Wincenty Skwarek \\ Rzeszów University of Technology, Faculty of Mechanical Engineering and Aeronautics, Rzeszów, Poland \\ e-mail: andrzejb@prz.edu.pl
}

\begin{abstract}
The work presents dynamics of a system of two wheeled mobile robots cooperating in the transport of large-size cargo in the form of a beam. The purpose of modeling of such a system was to obtain a mathematical model in an applicable form. Lagrange equations of the second type were used to describe dynamics, and then the projective method was used to eliminate Lagrange multipliers. Thanks to this approach, unknown dry friction forces at the contact points of robot wheels with the ground were eliminated from the description, and dynamics in controllable coordinates was obtained. In addition, the obtained model has structural properties that enable its use in synthesis of a control system based on the mathematical model.
\end{abstract}

Keywords: wheeled mobile robots, modeling of dynamics, formation robots

\section{Introduction}

A robot formation is a team of robots used to jointly perform a certain task. Robot formations are used where a given task is so complex or difficult that a single robot cannot perform it on its own. Such situations occur mainly in the case of large-size transport.

The construction of dynamical models of cooperating robots transporting an object is a current topic as evidenced by publications (Alipour et al., 2019). The paper (Abbaspour et al., 2015) presents a model of a system of three mobile robots connected by means of rotary joints to a large transported plate. The presented mathematical description using Newton Euler equations was applied in synthesis of the control algorithm.

Solutions to increase the implementation of wheeled robots in large-volume transport also include tractor-trailer systems. The tractor is an active unit containing drives and controls, while the trailer is a passive component articulated to the tractor. In modelling dynamical tasks of such non-holonomic systems, approaches are used both with the assumption of motion without any slip (Burghardt et al., 2020) and with the consideration of lateral and longitudinal wheel slip (Alipour et al., 2019).

The model solutions proposed in the article assume, similarly to the mathematical model of a single non-holonomic mobile robot, the lack of drive wheel slips. The modelling principles under such assumptions can be found in (Dhaouadi and Hatab, 2013; Yun and Sarkar, 1998). It should be noted that the assumption of rolling without any slip. The slip occurring is so small that it can be neglected when the velocities are small and there is sufficient friction between the wheels and the ground. It is clear that at higher speeds and accelerations and on a rough or slippery ground, the "pure rolling" assumption will not apply. There is a number of research papers explaining the effects of driving wheel slip and including descriptions of control algorithms 
for such objects (Wang and Low, 2008). Some researchers treat the slip as a disturbance and propose to compensate it using control algorithms (Yoo, 2012; Mohammadpour and Naraghi, 2011). Algorithms using elements of artificial intelligence such as fuzzy logic or artificial neural networks (Hendzel et al., 2013, 2015; Szuster et al., 2014; Hoang and Kang, 2016) work very well in this type of approach.

Mathematical models formulated for robot formations may serve for many purposes, such as research, design or control. Solving problems related to dynamics involves searching for cause-and-effect relationships between the forces in action and the torques being the causes of motion. The mathematical models can be used in analyses of their properties, identification and control.

This article presents a robot formation model that should be implementable for control purposes. Frequently, robot systems are characterised by a high degree of complexity with respect to movable components. Accounting for all of them significantly complicates calculations, negatively impacts transparency of the model, and makes it more difficult to implement it in control synthesis. In such situations, simplified models are used, which account only for such elements that have a significant impact on how the system actually behaves. Analytical mechanics provides for certain formalisms which can be used to describe dynamics of multi-agent non-holonomic systems. The formalisms that describe dynamics of non-holonomic systems, which are used most often, include Lagrange's equations, Maggi's equations (Giergiel and Żylski, 2005), Appell's equation (Burghardt, 2010) and Kane's equation (Tanner and Kyriakopoulos, 2001; Thanjavur and Rajagopalan, 1997).

The assumptions for the mathematical model of the formation analysed is that two identical wheeled robots are transporting a cylindrical object (a rod). Each robot consists of a frame and two drive wheels. Other elements have been omitted, assuming that they have a negligible impact on the dynamics of the whole system. This study aims at achieving a description of dynamics within controllable coordinates, as it would be of value due to its implementability in control synthesis. With such an aim, the study first uses Lagrange's equation, and then uses the projective method to convert the model so that it is described as a function of controllable coordinates.

\section{Kinematics of a formation of two robots transporting a bar}

This Section considers a case where two robots, moving on a flat $x y$ surface, are transporting a bar. The assumption is that the bar is attached above points $A_{1}$ and $A_{2}$, i.e. to midpoints of the line segments connecting the wheels (Fig. 1). The formation moves on a flat surface and the wheels are not subject to longitudinal or lateral slips. For the description of kinematics, let the generalised coordinate vector be $\mathbf{q}=\left[x_{M}, y_{M}, \varphi, \beta_{1}, \alpha_{11}, \alpha_{21}, \beta_{2}, \alpha_{12}, \alpha_{22}\right]^{\mathrm{T}}$ and the generalised velocity vector $\dot{\mathbf{q}}=\left[\dot{x}_{M}, \dot{y}_{M}, \dot{\varphi}, \dot{\beta}_{1}, \dot{\alpha}_{11}, \dot{\alpha}_{21}, \dot{\beta}_{2}, \dot{\alpha}_{12}, \dot{\alpha}_{22}\right]^{\mathrm{T}}$. The physical sense of the respective variables is as follows: $x_{M}, y_{M}$ are coordinates of a characteristic point of the bar transported within the $x y$ system; $\varphi$ - the angle of the bar rotation within the $x y$ plane; $\beta_{1}, \beta_{2}$ - the angles of rotation of the frames of robots 1 and $2 ; \alpha_{11}, \alpha_{21}$ - the angles of rotation of the wheels of robot $1 ; \alpha_{12}, \alpha_{22}$ - the angles of rotation of the wheels of robot 2 .

Given the geometry of the system, the kinematic equation is in a compact form of

$$
\mathbf{J}(\mathbf{q}) \dot{\mathbf{q}}=\mathbf{0}
$$

where the Jacobian matrix is as follows 


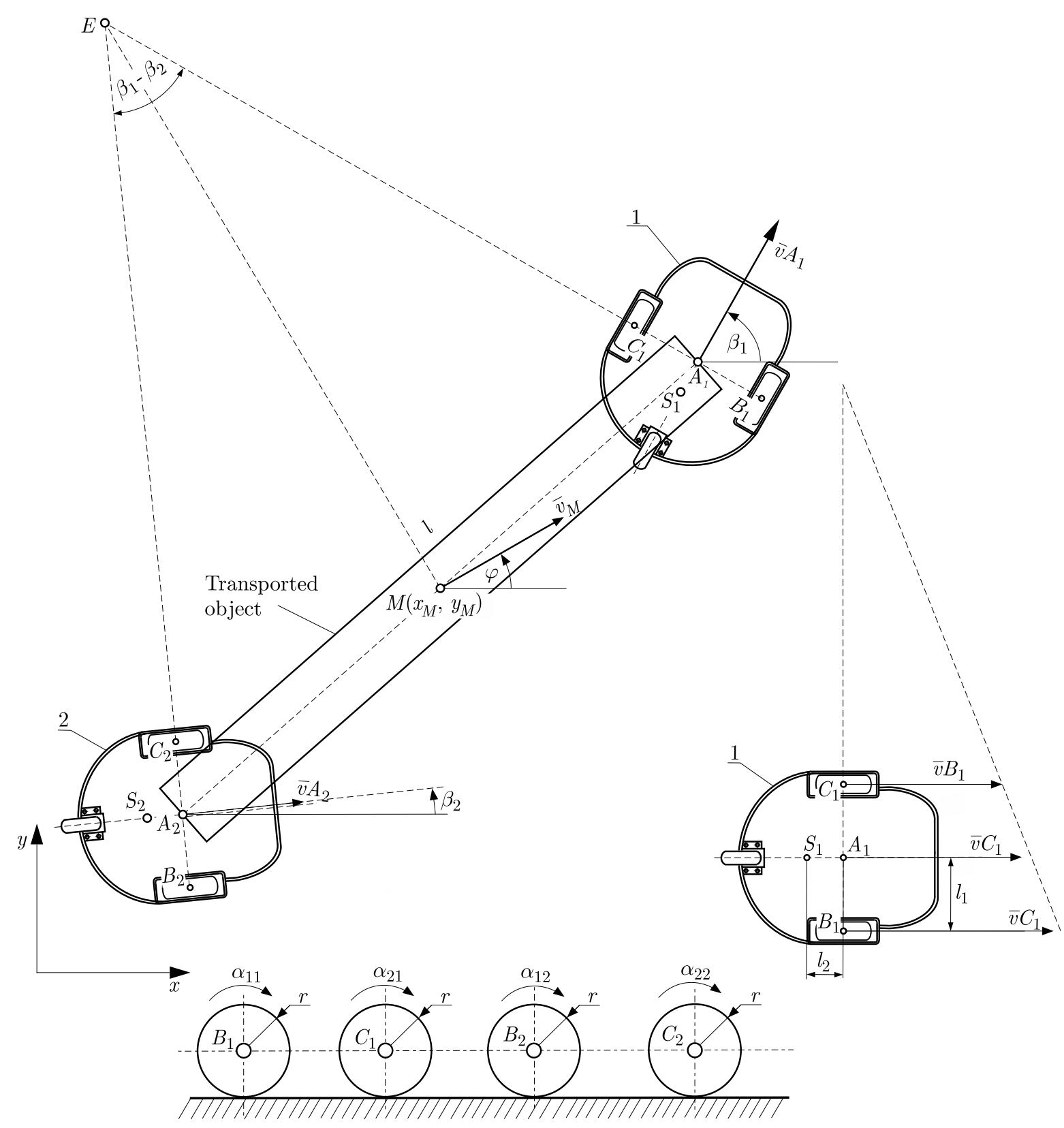

Fig. 1. Diagram of a robot formation transporting a bar

$$
\mathbf{J} \mathbf{q}=\left[\begin{array}{ccccccccc}
1 & 0 & 0 & 0 & -\frac{r}{4} \cos \beta_{1} & -\frac{r}{4} \cos \beta_{1} & 0 & -\frac{r}{4} \cos \beta_{2} & -\frac{r}{4} \cos \beta_{2} \\
0 & 1 & 0 & 0 & -\frac{r}{4} \sin \beta_{1} & -\frac{r}{4} \sin \beta_{1} & 0 & -\frac{r}{4} \sin \beta_{2} & -\frac{r}{4} \sin \beta_{2} \\
0 & 0 & 1 & -\frac{1}{2} & 0 & 0 & -\frac{1}{2} & 0 & 0 \\
0 & 0 & 0 & 1 & -\frac{r}{2 l_{1}} & \frac{r}{\left(2 l_{1}\right.} & 0 & 0 & 0 \\
0 & 0 & 0 & 0 & 0 & 0 & 1 & -\frac{r}{2 l_{1}} & \frac{r}{2 l_{1}}
\end{array}\right]
$$

where $r$ is the radius of the robots wheels and $l_{1}$ is the distance between the midpoint of the robot (point $A$ ) and the wheel. Out of 9 generalised coordinates, only four are controllable: the angle of rotation of the robot wheels, constituting a controllable coordinate vector $\mathbf{q}_{N}=\left[\alpha_{11}, \alpha_{21}, \alpha_{12}, \alpha_{22}\right]^{\mathrm{T}}$, which can be used to express kinematic constraint equations. is

The relationship between the generalised and controllable velocities $\dot{\mathbf{q}}_{N}=\left[\dot{\alpha}_{11}, \dot{\alpha}_{21}, \dot{\alpha}_{12}, \dot{\alpha}_{22}\right]^{\mathrm{T}}$

$$
\dot{\mathbf{q}}=\mathbf{D} \dot{\mathbf{q}}_{N}
$$


where the matrix $\mathbf{D}$ is as follows

$$
\mathbf{D}=\left[\begin{array}{cccc}
\frac{r \cos \beta_{1}}{4} & \frac{r \cos \beta_{1}}{4} & \frac{r \cos \beta_{2}}{4} & \frac{r \cos \beta_{2}}{4} \\
\frac{r \sin \beta_{1}}{4} & \frac{r \sin \beta_{1}}{4} & \frac{r \sin \beta_{2}}{4} & \frac{r \sin \beta_{2}}{4} \\
\frac{r}{4 l_{1}} & -\frac{r}{4 l_{1}} & \frac{r}{4 l_{1}} & -\frac{r}{4 l_{1}} \\
\frac{r}{2 l_{1}} & -\frac{r}{2 l_{1}} & 0 & 0 \\
1 & 0 & 0 & 0 \\
0 & 1 & 0 & 0 \\
0 & 0 & \frac{r}{2 l_{1}} & -\frac{r}{2 l_{1}} \\
0 & 0 & 1 & 0 \\
0 & 0 & 0 & 1
\end{array}\right]
$$

Matrices $\mathbf{J}$ and $\mathbf{D}$ satisfy $\mathbf{J D}=\mathbf{0}$, which allows us to remove the unknown dry friction forces in the description of dynamics (Abbaspour et al., 2015).

\section{Dynamics of a formation of two robots transporting a bar}

This Section presents a description of dynamics of the robot formation using Lagrange's equations of the second kind, which, in general notation, can be expressed as follows

$$
\frac{d}{d t}\left(\frac{\partial \mathbf{E}}{\partial \dot{\mathbf{q}}}\right)^{\mathrm{T}}-\left(\frac{\partial \mathbf{E}}{\partial \mathbf{q}}\right)^{\mathrm{T}}=\mathbf{Q}+\mathbf{J}^{\mathrm{T}}(\mathbf{q}) \boldsymbol{\lambda}
$$

where $\mathbf{E}$ is kinetic energy of the system, $\mathbf{Q}$ is the generalised force vector, and $\boldsymbol{\lambda}$ is the Lagrange multiplier vector. The right-hand side of Lagrange's equations is determined based on the principle of virtual work.

Virtual work $\delta L$ performed by the forces acting upon the robot formations is equal to the sum of virtual work performed by the forces acting upon the components thereof

$$
\delta L=\sum_{i=1}^{4} \mathbf{F} \dot{\mathbf{r}} d t+\sum_{j=1}^{4} \mathbf{M} \dot{\boldsymbol{\alpha}} d t
$$

The relationship in Eq. $(3,2)$ takes into account the sum of virtual works performed within translational motion by the force vector $\mathbf{F}$ and the sum of virtual works performed by the torque vector $\mathbf{M}$. Vectors $\dot{\mathbf{r}}$ and $\dot{\boldsymbol{\alpha}}$ are, respectively, the linear velocity vector of the respective components of the robot formation and the angular velocity vector thereof in rotational movement. External forces acting upon the system are shown in Fig. 2. Forces $P$ are loads applied to the relevant wheels, and forces $T$ are forces of dry friction between the wheels and the surface in the respective directions.

In the case analysed, the virtual work is

$$
\begin{aligned}
\delta L & =\left(M_{11}-N_{11} f_{11}\right) \delta \alpha_{11}+\left(M_{21}-N_{21} f_{21}\right) \delta \alpha_{21}+\left(M_{12}-N_{12} f_{12}\right) \delta \alpha_{12} \\
& +\left(M_{22}-N_{22} f_{22}\right) \delta \alpha_{22}
\end{aligned}
$$

where $M_{i j}$ is the torque driving the $i$-th wheel of the $j$-th robot, $N_{i j}$ is the pressure exerted on the $i$-th wheel of the $j$-th robot, $f_{i j}$ is the rolling friction factor of the $i$-th wheel of the $j$-th robot, and $\delta \alpha_{i j}$ is the virtual displacement of the $i$-th wheel of the $j$-th robot. The virtual work may also be expressed as

$$
\delta L=\sum_{k=1}^{n} Q_{k} \delta q_{k}
$$




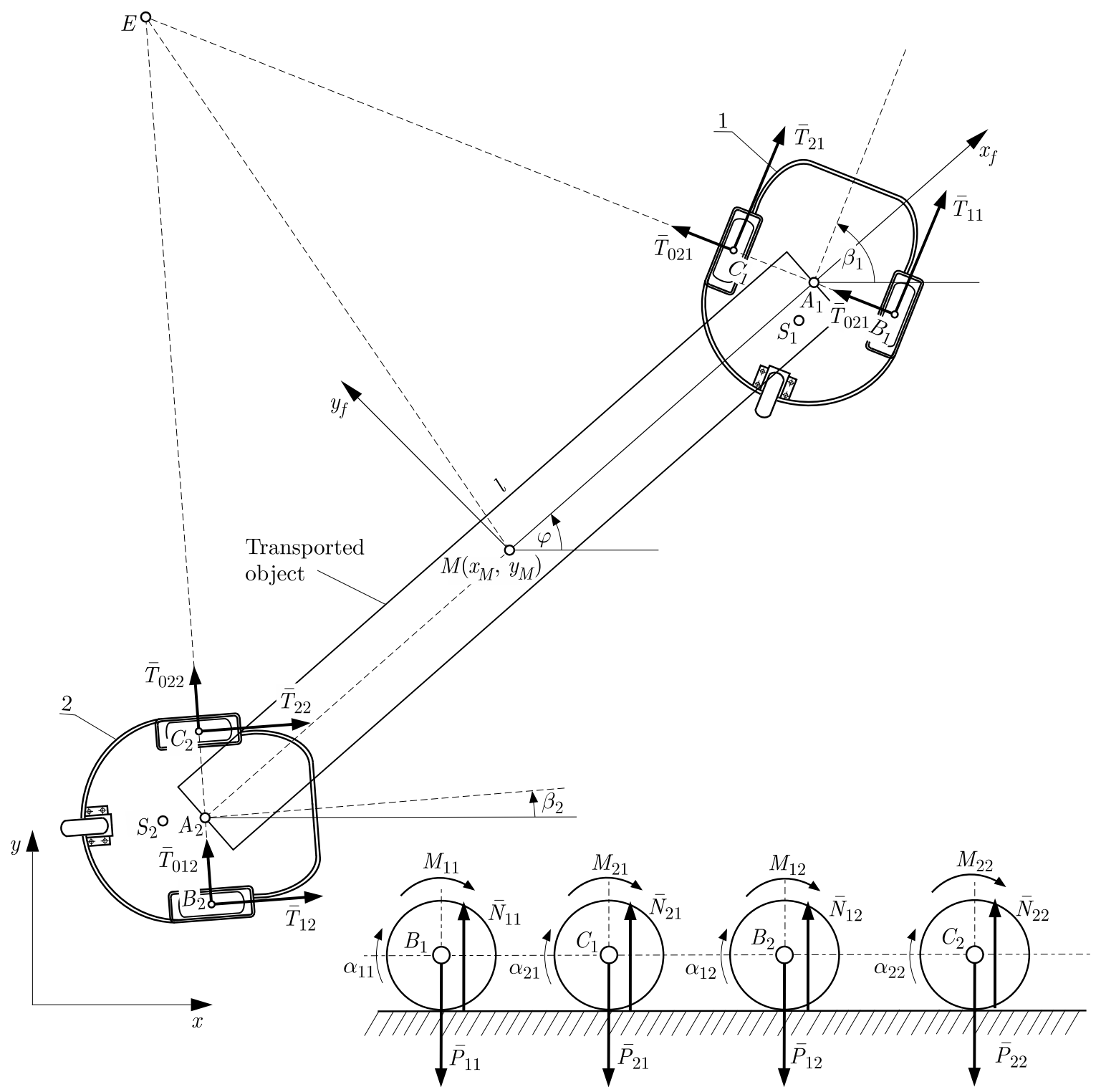

Fig. 2. Distribution of external forces acting upon the robot formation

where $Q_{k}$ is the $k$-generalised force, and $q_{k}$ is the $k$-generalised coordinate. Comparison of Eq. (3.3) with Eq. (3.4), taking into account the generalised coordinate vector, gives us the generalised force vector

$$
\mathbf{Q}=\left[\begin{array}{c}
0 \\
0 \\
0 \\
0 \\
M_{11}-N_{11} f_{11} \\
M_{21}-N_{21} f_{21} \\
0 \\
M_{12}-N_{12} f_{12} \\
M_{22}-N_{22} f_{22}
\end{array}\right]
$$

The Lagrange multiplier vector is expressed as

$$
\boldsymbol{\lambda}=\left[\lambda_{1}, \lambda_{2}, \lambda_{3}, \lambda_{4}, \lambda_{5}\right]^{\mathrm{T}}
$$


Taking into account the generalised force vector $\mathbf{Q}$, the Jacobian matrix $\mathbf{J}$ and the Lagrange multiplier vector $\boldsymbol{\lambda}$, the right-hand side of Lagrange's equations is expressed as

$$
\mathbf{Q}+\mathbf{J}^{\mathrm{T}}(\mathbf{q}) \boldsymbol{\lambda}=\left[\begin{array}{c}
\lambda_{1} \\
\lambda_{2} \\
\lambda_{3} \\
M_{11}-N_{11} f_{11}-\frac{r}{2} \lambda_{1} \cos \beta_{1}+\frac{r}{2} \lambda_{2} \sin \beta_{1}-\frac{r}{2 l_{1}} \lambda_{4} \\
M_{21}-N_{21} f_{21}+\frac{r}{2 l_{1}} \lambda_{4} \\
\frac{l_{1}}{2} \lambda_{1} \cos \beta_{1}+\frac{l_{1}}{2} \lambda_{1} \cos \beta_{2}+\frac{l_{1}}{2} \lambda_{2} \sin \beta_{2}-\frac{1}{2} \lambda_{3}+\lambda_{5} \\
M_{12}-N_{12} f_{12}-\frac{r}{2} \lambda_{1} \cos \beta_{2}-\frac{r}{2} \lambda_{2} \sin \beta_{2}-\frac{r}{2 l_{1}} \lambda_{5} \\
M_{22}-N_{22} f_{22}-\frac{r}{2 l_{1}} \lambda_{5}
\end{array}\right]
$$

The Lagrange multipliers represent forces of dry friction present in the plane of contact of the wheel with the surface. These values are not measurable. The analysis omits the resistance in the movement between the bar transported and the robots.

For the left-hand side of Lagrange's equations to be determined, it is necessary to calculate kinetic energy of the robot formation. The total kinetic energy equals the sum of kinetic energies of the robot respective components and the kinetic energy of the bar being transported. Within the model, this amounts to the sum of kinetic energies of the bar transported, robot frames, and the robot drive wheels. The kinetic energy of the support wheels and other components has been omitted due to its negligible impact. Figure 3 shows the system components and reference frames.

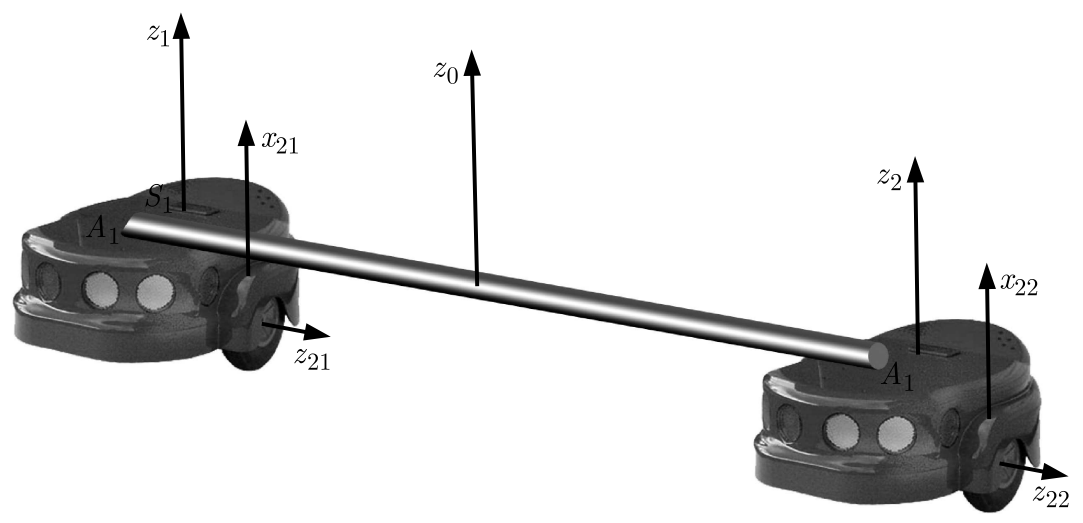

Fig. 3. Formation of two robots transporting a bar

The robot formation kinetic energy is given as the following relationship

$$
\begin{aligned}
E= & \left(\frac{1}{2} m_{0}+m_{1}+2 m_{11}\right) \dot{x}_{M}^{2}+\left(\frac{1}{2} m_{0}+m_{1}+2 m_{11}\right) \dot{y}_{M}^{2}+\left(\frac{1}{2} I_{0 z}+\frac{l^{2}}{4} m_{1}+\frac{l^{2}}{2} m_{11}\right) \dot{\varphi}^{2} \\
& +\left(\frac{1}{2} m_{1} l_{2}^{2}+m_{11} l_{1}^{2}+\frac{1}{2} I_{z 1}+I_{x 11}\right) \dot{\beta}_{1}^{2}+\frac{1}{2} I_{z 11} \dot{\alpha}_{11}^{2}+\frac{1}{2} I_{z 11} \dot{\alpha}_{21}^{2} \\
& +\left(\frac{1}{2} m_{1} l_{2}^{2}+m_{11} l_{1}^{2}+\frac{1}{2} I_{z 1}+I_{x 11}\right) \dot{\beta}_{2}^{2}+\frac{1}{2} I_{z 11} \dot{\alpha}_{12}^{2}+\frac{1}{2} I_{z 11} \dot{\alpha}_{22}^{2}+m_{1} l_{2} \sin \beta_{1} \dot{x}_{M} \dot{\beta}_{1} \\
& +m_{1} l_{2} \sin \beta_{2} \dot{x}_{M} \dot{\beta}_{2}+\left(-m_{1} l_{2} \cos \beta_{1}\right) \dot{y}_{M} \dot{\beta}_{1}+\left(-m_{1} l_{2} \cos \beta_{2}\right) \dot{y}_{M} \dot{\beta}_{2} \\
& +\left(-\frac{1}{2} m_{1} l_{2} l \cos \left(\varphi-\beta_{1}\right)\right) \dot{\beta}_{1} \dot{\varphi}+\frac{1}{2} m_{1} l_{2} l \cos \left(\varphi-\beta_{2}\right) \dot{\beta}_{2} \dot{\varphi}
\end{aligned}
$$

where $m_{0}$ is mass of the bar being transported, $m_{1}$ is mass of the robot, $m_{11}$ is mass of the robot wheel, $I_{0 z}$ is the bar moment of inertia about the axis $z$ intersecting the point $M, I_{z 1}$ is the robot 
moment of inertia about the axis going through its centre of mass, $I_{z 11}$ is the wheel moment of inertia about its rotation axis, $I_{x 11}$ is the wheel moment of inertia about the vertical axis going through its centre, $l$ is the bar length, and, finally, $l_{2}$ is the distance between the robot centre of mass and the point $A$. Equation (3.8) is based on the fact that the robots are identical, and thus it was assumed that $m_{1}=m_{2}, m_{11}=m_{21}=m_{12}=m_{22}, I_{z 1}=I_{z 2}, I_{z 11}=I_{z 21}=I_{z 1} 2=I_{z 22}$, $I_{x 11}=I_{x 21}=I_{x 12}=I_{x 22}$.

In matrix notation, the left-hand sides of Lagrange's equations are

$$
\frac{d}{d t}\left(\frac{\partial \mathbf{E}}{\partial \dot{\mathbf{q}}}\right)^{\mathrm{T}}-\left(\frac{\partial \mathbf{E}}{\partial \mathbf{q}}\right)^{\mathrm{T}}=\mathbf{M}(\mathbf{q}) \ddot{\mathbf{q}}+\mathbf{C}(\mathbf{q}, \dot{\mathbf{q}}) \dot{\mathbf{q}}
$$

where $\mathbf{M}$ is the inertia matrix, and $\mathbf{C}(\mathbf{q}, \dot{\mathbf{q}}) \dot{\mathbf{q}}$ is the vector of centrifugal and Coriolis forces. The inertia matrix is in the form of a symmetric matrix

$$
\mathbf{M}=\left[\begin{array}{ccccccccc}
M_{11} & 0 & 0 & M_{14} & 0 & 0 & M_{17} & 0 & 0 \\
0 & M_{22} & 0 & M_{24} & 0 & 0 & M_{27} & 0 & 0 \\
0 & 0 & M_{33} & M_{34} & 0 & 0 & M_{37} & 0 & 0 \\
M_{41} & M_{42} & M_{43} & M_{44} & 0 & 0 & 0 & 0 & 0 \\
0 & 0 & 0 & 0 & I_{z 11} & 0 & 0 & 0 & 0 \\
0 & 0 & 0 & 0 & 0 & I_{z 11} & 0 & 0 & 0 \\
M_{71} & M_{72} & M_{73} & 0 & 0 & 0 & M_{77} & 0 & 0 \\
0 & 0 & 0 & 0 & 0 & 0 & 0 & I_{z 11} & 0 \\
0 & 0 & 0 & 0 & 0 & 0 & 0 & 0 & I_{z 11}
\end{array}\right]
$$

where

$$
\begin{aligned}
& M_{11}=m_{0}+2 m_{1}+4 m_{11} \\
& M_{41}=M_{14}=m_{1} l_{2} \sin \beta_{1} \\
& M_{71}=M_{17}=m_{1} l_{2} \sin \beta_{2} \\
& M_{22}=m_{0}+2 m_{1}+4 m_{11} \\
& M_{42}=M_{24}=-m_{1} l_{2} \cos \beta_{1} \\
& M_{72}=M_{27}=-m_{1} l_{2} \cos \beta_{2} \\
& M_{33}=I_{0 z}+\frac{l^{2}}{2} m_{1}+m_{11} l^{2} \\
& M_{43}=M_{34}=-\frac{1}{2} m_{1} l_{2} l \cos \left(\varphi-\beta_{1}\right) \\
& M_{73}=M_{37}=\frac{1}{2} m_{1} l_{2} l \cos \left(\varphi-\beta_{2}\right) \\
& \left.M_{44}=M_{77}=m_{1} l_{2}^{2}+2 m_{11} l_{1}^{2}+I_{z 1}+2 I_{x 11}\right)
\end{aligned}
$$

The matrix $\mathbf{C}(\mathbf{q}, \dot{\mathbf{q}})$ has the form of

$$
\mathbf{C}(\mathbf{q}, \dot{\mathbf{q}})=\left[\begin{array}{ccccccccc}
0 & 0 & 0 & m_{1} l_{2} \cos \beta_{1} \dot{\beta}_{1} & 0 & 0 & m_{1} l_{2} \cos \beta_{2} \dot{\beta}_{2} & 0 & 0 \\
0 & 0 & 0 & m_{1} l_{2} \sin \beta_{1} \dot{\beta}_{1} & 0 & 0 & m_{1} l_{2} \sin \beta_{2} \dot{\beta}_{2} & 0 & 0 \\
0 & 0 & 0 & -a \dot{\beta}_{1} & 0 & 0 & b \dot{\beta}_{2} & 0 & 0 \\
0 & 0 & a \dot{\varphi} & 0 & 0 & 0 & 0 & 0 & 0 \\
0 & 0 & 0 & 0 & 0 & 0 & 0 & 0 & 0 \\
0 & 0 & 0 & 0 & 0 & 0 & 0 & 0 & 0 \\
0 & 0 & -b \dot{\varphi} & 0 & 0 & 0 & 0 & 0 & 0 \\
0 & 0 & 0 & 0 & 0 & 0 & 0 & 0 & 0 \\
0 & 0 & 0 & 0 & 0 & 0 & 0 & 0 & 0
\end{array}\right]
$$


where $a=\left[m_{1} l_{2} l \sin \left(\varphi-\beta_{1}\right)\right] / 2, b=\left[m_{1} l_{2} l \sin \left(\varphi-\beta_{2}\right)\right] / 2$. Ultimately, the dynamics of the robot formation in generalised coordinates is specified as

$$
\mathbf{M}(\mathbf{q}) \dot{\mathbf{q}}+\mathbf{C}(\mathbf{q}, \dot{\mathbf{q}}) \dot{\mathbf{q}}=\mathbf{Q}+\mathbf{J}^{\mathrm{T}}(\mathbf{q}) \boldsymbol{\lambda}
$$

The model is characterised by the fact that the matrix $\mathbf{S}=\dot{\mathbf{M}}-2 \mathbf{C}$ is skew-symmetric, which is a key property from the point of view of control synthesis of the system analysed with the use of methods that implement mathematical models.

\section{Dynamics of the robot formation in controllable coordinates}

This Section deals with the conversion of description concerning dynamics of the robot formation into description of dynamics in controllable coordinates. The transformation between generalised velocities and controllable velocities is represented by Eq. (2.3). Based on this equation, the following generalised acceleration has been drawn

$$
\ddot{\mathbf{q}}=\mathbf{D} \ddot{\mathbf{q}}_{N}+\dot{\mathbf{D}} \dot{\mathbf{q}}_{N}
$$

By substituting Eqs. (2.3) and (4.1) into Eq. (3.13), we arrive at the equation (Blajer, 1998)

$$
\mathbf{M D} \ddot{\mathbf{q}}_{N}+(\mathbf{M D}+\mathbf{C D}) \dot{\mathbf{q}}_{N}=\mathbf{Q}+\mathbf{J}^{\mathrm{T}}(\mathbf{q}) \boldsymbol{\lambda}
$$

which, when multiplied on the left-hand side by $\mathbf{D}^{\mathrm{T}}$, is expressed as

$$
\mathbf{D}^{\mathrm{T}} \mathbf{M D} \ddot{\mathbf{q}}_{N}+\mathbf{D}^{\mathrm{T}}(\mathbf{M} \dot{\mathbf{D}}+\mathbf{C D}) \dot{\mathbf{q}}_{N}=\mathbf{D}^{\mathrm{T}} \mathbf{Q}+\mathbf{D}^{\mathrm{T}} \mathbf{J}^{\mathrm{T}}(\mathbf{q}) \boldsymbol{\lambda}
$$

By assuming $\mathbf{D}^{\mathrm{T}} \mathbf{M D}=\mathbf{M}_{N}, \mathbf{D}^{\mathrm{T}}(\mathbf{M} \dot{\mathbf{D}}+\mathbf{C D})=\mathbf{C}_{N}$ and $\mathbf{D}^{\mathrm{T}} \mathbf{Q}=\mathbf{Q}_{N}$, the equation concerning the dynamics in controlled coordinates is finally expressed as

$$
\mathbf{M}_{N} \ddot{\mathbf{q}}_{N}+\mathbf{C}_{N} \dot{\mathbf{q}}_{N}=\mathbf{Q}_{N}
$$

where $\mathbf{D}^{\mathrm{T}} \mathbf{J}^{\mathrm{T}}=\mathbf{J D}=\mathbf{0}$. This way, the Lagrange multiplier vector $\boldsymbol{\lambda}$, which contains variables that cannot be measured, has been removed. A key feature of the model is the skew-symmetric nature of the matrix $\mathbf{S}_{N}=\dot{\mathbf{M}}_{N}-2 \mathbf{C}_{N}$. To prove this property, the matrix $\mathbf{S}_{N}$ expressed as

$$
\begin{aligned}
\mathbf{S}_{N} & =\dot{\mathbf{M}}_{N}-2 \mathbf{C}_{N}=\frac{d}{d t}\left(\mathbf{D}^{\mathrm{T}} \mathbf{M D}\right)-2 \mathbf{D}^{\mathrm{T}}(\mathbf{M} \dot{\mathbf{D}}+\mathbf{C D})=\frac{d}{d t}\left(\mathbf{D}^{\mathrm{T}}\right) \mathbf{M D}+\mathbf{D}^{\mathrm{T}} \dot{\mathbf{M}} \mathbf{D} \\
& +\mathbf{D}^{\mathrm{T}} \mathbf{M} \dot{\mathbf{D}}-2 \mathbf{D}^{\mathrm{T}}(\mathbf{M} \dot{\mathbf{D}}+\mathbf{C D})=\mathbf{D}^{\mathrm{T}}(\dot{\mathbf{M}}-2 \mathbf{C}) \mathbf{D}=\mathbf{D}^{\mathrm{T}} \mathbf{S} \mathbf{D}
\end{aligned}
$$

where the following property was used: $\frac{d}{d t}\left(\mathbf{D}^{\mathrm{T}}\right) \mathbf{M D}=\mathbf{D}^{\mathrm{T}} \mathbf{M} \dot{\mathbf{D}}$. Multiplying the skew-symmetric matrix $\mathbf{S}$ on the left-hand side by $\mathbf{D}^{\mathrm{T}}$ and on the right-hand side by $\mathbf{D}$ yields a skew-symmetric matrix as a result. Therefore, the matrix $\mathbf{S}_{N}$ is indeed skew-symmetrical.

\section{Simulation}

Simulations of the dynamics task were carried out in Matlab/Simulink using the work emulator described in publications (Burghardt, 2008; Kurc et al., 2016). For the purpose of simulation it was assumed that the $M$ point of the transported beam moves along the track shown in Fig. 4c with an assumed linear velocity as in Fig. 4a and an angular velocity as in Fig. 4b. It was assumed that the waveforms describing the angular and linear velocity profiles are C2 class functions. 
(a)

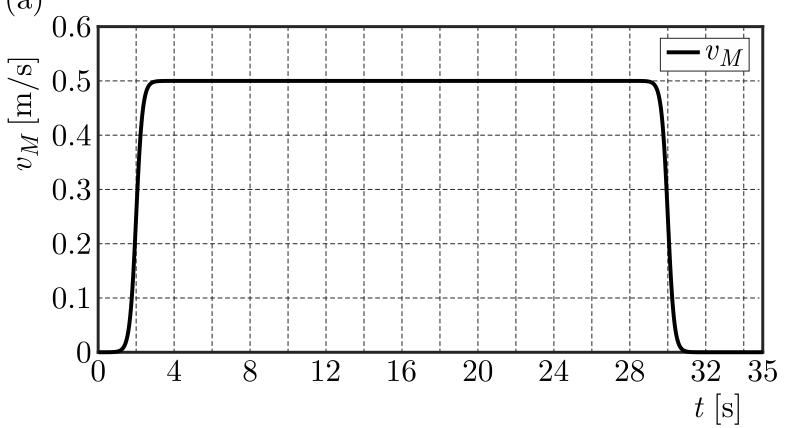

(c)

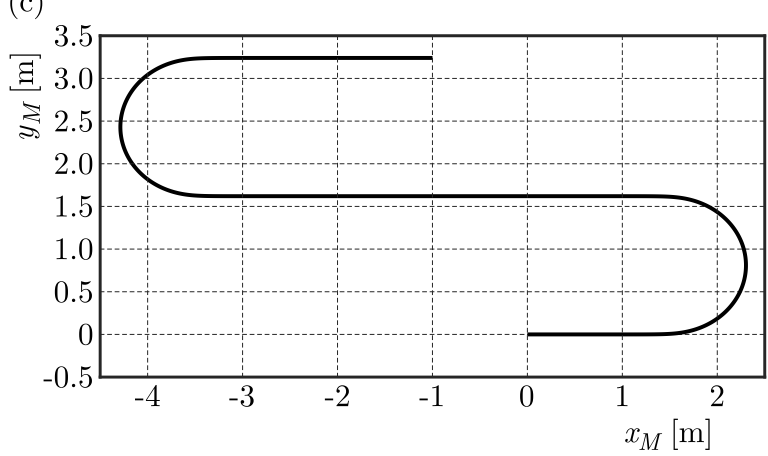

(b)

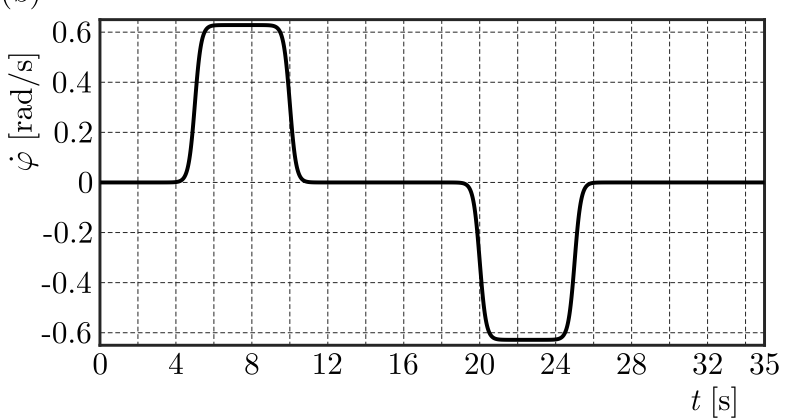

(d)

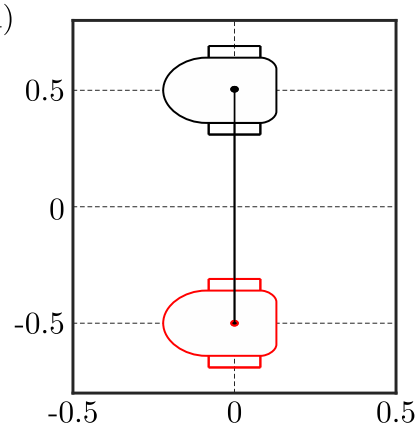

Fig. 4. (a) Velocity profile of the $M$ point, (b) angular velocity of the transported beam, (c) generated motion path of the $M$ point, (d) initial setting of the robots and the beam in the emulator

(a)

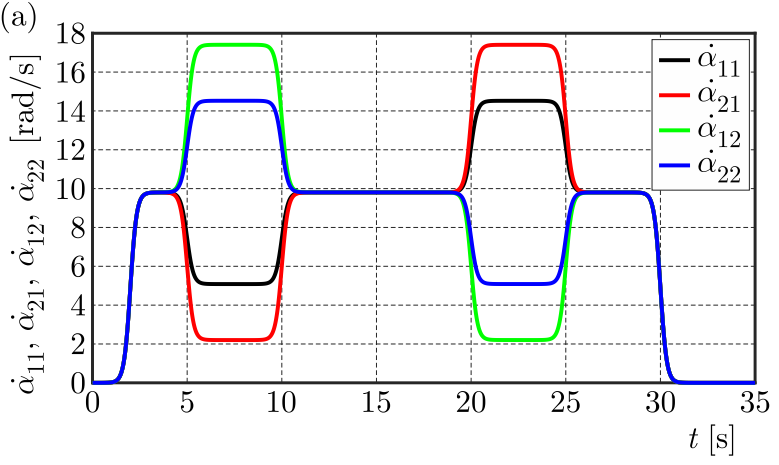

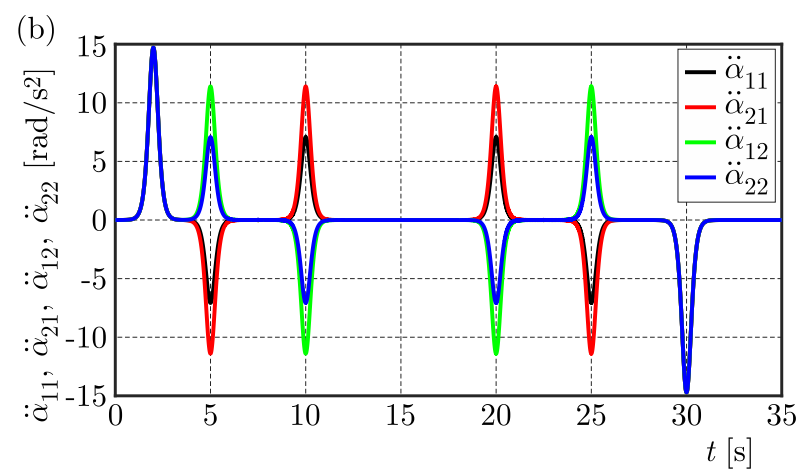

Fig. 5. (a) Angular velocities of the robot driving wheels, (b) angular accelerations of the driving wheels

For the assumed trajectories and initial setting of the robots (Fig. 4d), the angular velocities of the driving wheels, shown in Fig. 5a, and the angular accelerations of the driving wheels, shown in Fig. 5b, were generated based on the kinematic relations described in works (Burghardt, 2010).

The obtained values of velocities and accelerations are the input parameters for simulation of the correctness of solving the simple dynamics task described by dependency (4.4) where values of elements of the $\mathbf{M}$ matrix, $\mathbf{C}_{N}$ matrix and $\mathbf{Q}_{N}$ vector were calculated based on Section 3 and 4. As a result of the simulation, the time waveforms of the torques driving the wheels of two mobile robots performing the task of transporting a beam along the track shown in Fig. 4c were obtained. A view showing the shape of the robot formation in successive time intervals, presented in Fig. 6b, was generated with the use of the robot work emulator. The frequency of generating successive images by the emulator is $2 \mathrm{~Hz}$.

During the task, the mass of the transported solid was assumed as $10 \mathrm{~kg}$. The appearance of the task and the shape of the time waveforms of the driving torques confirm the correctness of the dependence obtained in Section 4. 
(a)

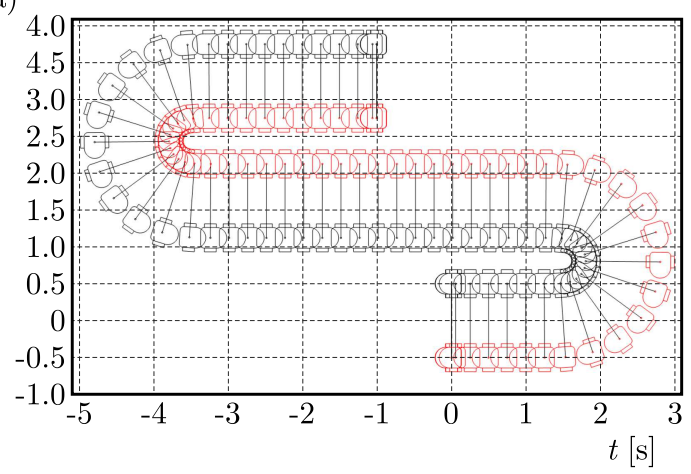

(b)

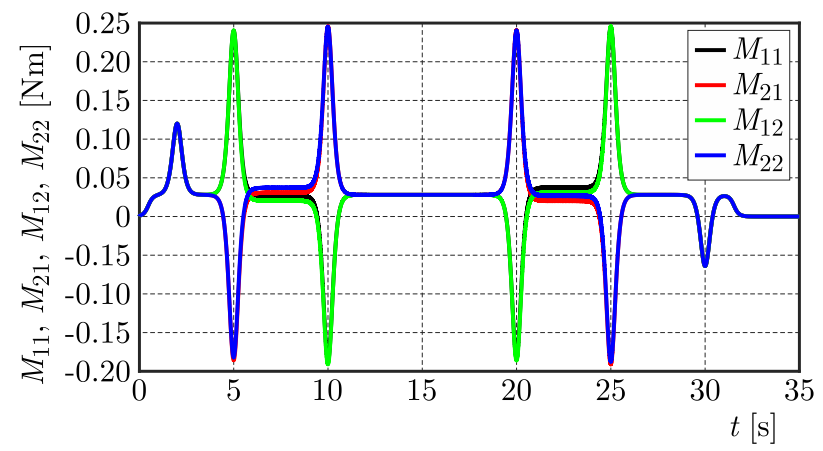

Fig. 6. (a) The course of the transportation task, (b) solution of the inverse dynamics task

\section{Conclusions}

The study presents the model of dynamics of a robot formation transporting a certain load. The model obtained accounts for the most significant phenomena related to the system motion. The projective method allowed Lagrange multipliers to be removed from the description. The lack of Lagrange multipliers and the skew-symmetrical nature of the matrix $\mathbf{S}_{N}=\dot{\mathbf{M}}_{N}-2 \mathbf{C}_{N}$ makes the model implementable in control synthesis using methods that implement mathematical models.

The mathematical description of the dynamics of the system of two robots transporting a beam was simulated in Matlab/Simulink. The use of the author's emulator of robot operation allowed visualization of the system motion under simulation conditions. The obtained time waveforms of the torques driving the wheels of the robots and the appearance of motion of the robots during the simulation confirm the correctness of the obtained solutions.

\section{References}

1. Abbaspour A., Alipour K., Jafari H.Z., Moosavian S.A., 2015, Optimal formation and control of cooperative wheeled mobile robots, Comptes Rendus Mécanique, 343, 5-6, 307-321

2. Alipour K., Robat A.B., Tarvirdizadeh B., 2019, Dynamics modeling and sliding mode control of tractor-trailer wheeled mobile robots subject to wheels slip, Mechanism and Machine Theory, 138, 16-37

3. Blajer W., 1998, Methods of Dynamics of Multi-Member Systems (in Polish), Politechnika Radomska, Radom

4. Burghardt A., 2008, Proposal for a rapid prototyping environment for algorithms intended for autonomous mobile robot control, Mechanics and Mechanical Engineering, 1, 5-16

5. Burghardt A., 2010, Modelling of dynamics of a wheeled robot by Appell's equation (in Polish), Acta Mechanica et Automatica, 4, 9-12

6. Burghardt A., Szybicki D., Kurc K., Muszynska M., 2020, Mechatronic designing and prototyping of a mobile wheeled robot driven by a microcontroller, Journal of Theoretical and Applied Mechanics, 58, 1, 127-142

7. Dhaouadi R., Hatab A., 2013, Dynamic modelling of differential-drive mobile robots using Lagrange and Newton-Euler methodologies: a unified framework, Advances in Robotics and Automation, 2, 2, 1-7

8. Giergiel J., ŻYlski W., 2005, Description of motion of a mobile robot by Maggie's equations, Journal of Theoretical and Applied Mechanics, 43, 3, 511-521 
9. Hendzel Z., Burghardt A., Szuster M., 2013, Adaptive critic designs in control of robots formation in unknown environment, Proceedings of International Conference on Artificial Intelligence and Soft Computing, ICAISC 2013, DOI:10.1007/978-3-642-38610-7_33, 351-362

10. Hendzel Z., Burghardt A., Szuster M., 2015, Artificial intelligence algorithms in behavioural control of wheeled mobile robots formation, [In:] Computational Intelligence. Studies in Computational Intelligence, V. 577, Madani K., Correia A., Rosa A., Filipe J. (Eds), Springer, Cham

11. HoAng N.B., KAng H.J., 2016, Neural network-based adaptive tracking control of mobile robots in the presence of wheel slip and external disturbance force, Neurocomputing, 188, 12-22

12. Kurc K., Szybicki D., Burghardt A., Muszyńska M., 2016, The application of virtual prototyping methods to determine the dynamic parameters of mobile robot, Open Engineering, 6, 1, 55-63

13. Mohammadpour E., Naraghi M., 2011, Robust adaptive stabilization of skid steer wheeled mobile robots considering slipping effects, Advanced Robotics, 25, 1-2, 205-227

14. Szuster M., Hendzel Z., Burghardt A., 2014, Fuzzy sensor-based navigation with neural tracking control of the wheeled mobile robot, Artificial Intelligence and Soft Computing, Lecture Notes in Computer Science, Springer Verlag, 8468, 302-313

15. Tanner H.G., Kyriakopoulos K.J., 2001, Mobile manipulator modeling with Kane's approach, Robotica, 19, 6, 675-690

16. Thanjavur K., Rajagopalan R., 1997, Ease of dynamic modelling of wheeled mobile robots (WMRs) using Kane's approach, Proceedings of International Conference on Robotics and Automation, 4, IEEE, 2926-2931

17. WANG D., Low C.B., 2008, Modeling and analysis of skidding and slipping in wheeled mobile robots: control design perspective, IEEE Transactions on Robotics, 24, 3, 676-687

18. Yoo S.J., 2012, Approximation-based adaptive control for a class of mobile robots with unknown skidding and slipping, International Journal of Control, Automation, and Systems, 10, 4, 703-710

19. Yun X., SARKAR N., 1998, Unified formulation of robotic systems with holonomic and nonholonomic constraints, IEEE Transactions on Robotics and Automation, 14, 4, 640-650

Manuscript received April 17, 2021; accepted for print August 4, 2021 